\title{
Circular RNA circTRIM33-12 acts as the sponge of MicroRNA-191 to suppress hepatocellular carcinoma progression
}

\author{
Peng-Fei Zhang ${ }^{1 \dagger}$, Chuan-Yuan Wei ${ }^{1 \dagger}$, Xiao-Yong Huang ${ }^{1 \dagger}$, Rui Peng ${ }^{1 \dagger}$, Xuan Yang ${ }^{1}$, Jia-Cheng Lu', Chi Zhang ${ }^{1}$, \\ Chao Gao', Jia-Bin Cai ${ }^{1}$, Ping-Ting Gao ${ }^{1}$, Dong-Mei Gao ${ }^{1}$, Guo-Ming Shi ${ }^{1 *}$, Ai-Wu Ke ${ }^{1 *}$ (D) and Jia Fan ${ }^{1,2^{*}}$
}

\begin{abstract}
Background: Recently, the dysregulation of circular RNA (circRNA) have been shown to have important regulatory roles in cancer development and progression, including hepatocellular carcinoma (HCC). However, the roles of most circRNAs in HCC are still unknown.

Methods: The expression of circular tripartite motif containing 33-12 (circTRIM33-12) in HCC tissues and cell lines was detected by qRT-PCR. The role of circTRIM33-12 in HCC progression was assessed by western blotting, CCK-8, flow cytometry, transwell and a subcutaneous tumor mouse assays both in vitro and in vivo. In vivo circRNA precipitation, RNA immunoprecipitation, luciferase reporter assays were performed to evaluate the interaction between circTRIM33-12 and miR-191.

Results: Here, we found that circTRIM33-12, is downregulated in HCC tissues and cell lines. The downregulation of circTRIM33-12 in HCC was significantly correlated with malignant characteristics and served as an independent risk factor for the overall survival (OS) and recurrence-free survival (RFS) of patients with HCC after surgery. The reduced expression of circTRIM33-12 in HCC cells increases tumor proliferation, migration, invasion and immune evasion. Mechanistically, we demonstrated that circTRIM33-12 upregulated TET1 expression by sponging miR-191, resulting in significantly reduced 5 -hydroxymethylcytosine $(5 \mathrm{hmC})$ levels in HCC cells.

Conclusions: These results reveal the important role of circTRIM33-12 in the proliferation, migration, invasion and immune evasion abilities of HCC cells and provide a new perspective on circRNAs in HCC progression.
\end{abstract}

Keywords: Hepatocellular carcinoma, Circular RNA, TET1, Histone methylation

\section{Background}

Hepatocellular carcinoma $(\mathrm{HCC})$ is the third leading cause of cancer-related deaths worldwide [1]. HCC is clinically characterized by its invasiveness, poor prognosis and limited therapeutic opportunities. At present, surgery is the most common form of therapy for HCC, but the characteristics of multifocal development and distant metastases preclude surgical treatment from being curative in most

\footnotetext{
*Correspondence: shi.guoming@zs-hospital.sh.cn; keaiwu_fd@hotmail.com; fan.ji@@zs-hospital.sh.cn

${ }^{\dagger}$ Peng-Fei Zhang, Chuan-Yuan Wei, Xiao-Yong Huang and Rui Peng contributed equally to this work.

${ }^{1}$ Liver Cancer Institute, Zhongshan Hospital, Key Laboratory of Carcinogenesis and Cancer Invasion, Fudan University, Ministry of Education, 180 Fenglin

Road, Shanghai 200032, People's Republic of China

Full list of author information is available at the end of the article
}

HCC cases [2]; thus, the further understanding of the molecular mechanisms underlying $\mathrm{HCC}$ progression is of paramount importance.

Growing evidence has indicated that the dysregulation of circular RNAs (circRNAs) and DNA methylation contribute to tumorigenesis and progression [3-5]. circRNAs are highly conserved and are characterized as covalently closed loop structures with neither a $5^{\prime}$ to $3^{\prime}$ polarity nor a polyadenylated tail; they are RNA transcripts generated by the back-splicing of a single pre-mRNA and have generegulatory potential $[6,7]$. Recently, studies have revealed that circRNAs were abnormally expressed in several cancer tissues and correlated with disease progression and prognosis [8]. The critical physiological functions of circRNAs include miRNA sponges, protein translation

(C) The Author(s). 2019 Open Access This article is distributed under the terms of the Creative Commons Attribution 4.0 International License (http://creativecommons.org/licenses/by/4.0/), which permits unrestricted use, distribution, and 
templates and the regulation of parental expression [9-11]. Aberrant circRNA expression also plays an essential role in HCC tumorigenesis and progression [12-14]. The ten eleven translocation (TET) family of methylcytosine dioxygenases (including TET1, TET2, and TET3) participated in converting 5-methylcytosine $(5 \mathrm{mC})$ to 5 hydroxymethylcytosine $(5 \mathrm{hmC})$, and participated in removing existing DNA methylation tags in cells [15-17]. It has been reported that reduced $5 \mathrm{hmC} / \mathrm{TET} 1$ expression is associated with the progression of $\mathrm{HCC}$ and that increased $5 \mathrm{mC}$ expression positively correlates with $\mathrm{HCC}$ invasion and recurrence; this suggests that the DNA methylation/ demethylation regulated by the TET family of methylcytosine dioxygenases, may participate in the process of HCC progression $[18,19]$.

Here, we analyzed the expression profiles of tripartite motifs containing 33 (TRIM33)-derived circRNAs in HCC and paired adjacent normal tissues (ANTs) using qRTPCR. The expression levels of circTRIM33-12 were significantly reduced in HCC tissues compared with the expression levels in ANTs. Kaplan-Meier survival analysis showed that reduced circTRIM33-12 levels correlated with a poorer survival of HCC patients. We examined the functions of circTRIM33-12 in HCC and found that knockdown of circTRIM33-12 promoted cell proliferation, invasion, and immune evasion. In addition, we found that circTRIM33-12 may function as the sponge of oncogenic miR-191 to upregulate TET1 expression and to consequently suppress HCC progression. Therefore, reduced TRIM33-12 expression may serve as a promising biomarker for prognosis prediction and as a potential therapeutic target for HCC patients.

\section{Methods}

\section{Cell lines and clinical tissues}

The seven HCC cell lines used in this study; and the types of tissue samples collected are described in the Additional file 1: Supplementary Materials and Methods.

\section{Quantitative real-time polymerase chain reaction analysis,} western blotting analysis, and immunofluorescence assays

Quantitative real-time polymerase chain reaction (qRT$\mathrm{PCR}$ ), immunofluorescence, and western blotting analyses were performed as described in the references and described in the Additional file 1: Supplementary Materials and Methods [20, 21]. The primers and antibodies used in this study are listed in Additional file 2: Table S1 and Additional file 3: Table S2.

\section{Transfection experiment}

The transfection experiments are described in the Additional file 1: Supplementary Materials and Methods.
The target sequences of shRNAs are listed in Additional file 4: Table S3.

\section{Immunohistochemistry}

Immunohistochemistry was performed as described in our previous studies [21, 22]. An anti-NKG2D antibody was used to detect the expression of NKG2D. The antibodies used in this study are listed in Additional file 3: Table S2. The intensity of the positive staining was measured as described in the Additional file 1: Supplementary Materials and Methods [20].

\section{In vivo circRNA precipitation}

Biotin-labeled circTRIM33-12 and negative control probes (Additional file 5: Tables S4) were synthesized by Sangon Biotech. The in vivo circRNA immunoprecipitation (circRIP) assay was performed as described in the references and is described in the Additional file 1: Supplementary Materials and Methods [12].

\section{Knockdown or overexpression of circTRIM33-12}

Small hairpin RNAs (shRNAs) of circTRIM33-12 and circTRIM33-12-overexpressing plasmids were synthesized by GenePharma (Shanghai, China), and the shRNA targeting the junction region of the circTRIM33-12 sequence was also synthesized. Huh 7 and SMMC-7721 cells were transfected with circTRIM33-12 shRNA or the overexpressing plasmids using Lipofectamine 2000 (Invitrogen, Carlsbad, CA, USA) according to the manufacturer's instructions.

\section{Cell proliferation, migration, and Matrigel invasion assay} Cell proliferation, migration, and Matrigel invasion assays were performed as described in the Additional file 1: Supplementary Materials and Methods [23].

\section{Flow cytometry assay of the cell cycle}

Cells were fixed in $70 \%$ ethanol overnight at $4{ }^{\circ} \mathrm{C}$. Then, the fixed cells were resuspended in staining solution (Beyotime, Shanghai) and were incubated for $30 \mathrm{~min}$ at $4{ }^{\circ} \mathrm{C}$. Finally, the stained cells were measured by flow cytometry (Beckman Coulter).

\section{RNA immunoprecipitation (RIP)}

RNA immunoprecipitation (RIP) assays were performed using a Magna RIP RNABinding Protein Immunoprecipitation Kit (Millipore) according to the manufacturer's instructions. The argonaute 2 (AGO2) and IgG antibodies used in this study are listed in Additional file 3: Table S2.

\section{Luciferase reporter assay}

The mutant luciferase reporter vectors were generated using a Mutagenesis Kit (QIAGEN, California, USA) according to the manufacturer's instructions. Huh 7 cells 
were seeded into 96-well plates and were cotransfected with a luciferase reporter vector and miR-191 mimics or the negative control using the Lipofectamine 2000 transfection reagent. After $48 \mathrm{~h}$, the firefly and Renilla luciferase activities were quantified with a dual-luciferase reporter assay (Promega, USA).

\section{In vivo tumor growth and metastasis assays}

The in vivo tumor growth and metastasis assays are described in the Additional file 1: Supplementary Materials and Methods.

\section{Statistical analysis}

Statistical analysis was performed with SPSS software (19.0; SPSS, Inc., Chicago, IL) as previously described in reference [22]. In brief, the values are expressed as the mean \pm standard deviation (SD). Student's $t$ test was used for comparisons between groups. The categorical data were analyzed by chi-square or Fisher's exact tests. Correlation analysis was performed among circTRIM33, TET1, and NKG2D. The cumulative recurrence and survival rates were analyzed using Kaplan-Meier's method and the log-rank test. Cox's proportional hazard regression model was used to analyze independent prognostic factors. $P<0.05$ was considered statistically significant.

\section{Results}

Dysregulated TRIM33-derived circTRIM33-12 in HCC

TRIM33 is a tumor suppressor that can inhibit tumor cell progression and tumorigenesis by different mechanisms in several cancers, including HCC [24-26]. A previous study has reported that the downregulation of TRIM33 in HCC was caused by the hypermethylation of CpG islands in the TRIM33 promoter [24], indicating that circRNA-derived TRIM33 expression might be downregulated in HCC cells. Therefore, we speculated that the possibility of circRNA-derived TRIM33 acts as a tumor suppressor in HCC cells. To investigate TRIM33derived circRNA expression in HCC tissues, we analyzed four pairs of HCC tissue samples (four HCC tissues and matched adjacent nontumor liver tissues) by using qRTPCR. Among 18 circRNAs, we found that circTRIM3312 (hsa_circRNA8662-12) expression was significantly and consistently reduced in four of the HCC tumor tissues compared to that in the matched adjacent nontumor liver tissues (Fig. 1a). Thus, we focused on the expression and role of circTRIM33-12 (circRNA derived from exons 12 and 18 of the TRIM33) for further study (Fig. 1b). To further investigate the prognostic significance of circTRIM33-12 expression in HCC patients, the levels of circTRIM33-12 were quantified in a cohort of $200 \mathrm{HCC}$ and adjacent tissues using qRT-PCR (Fig.

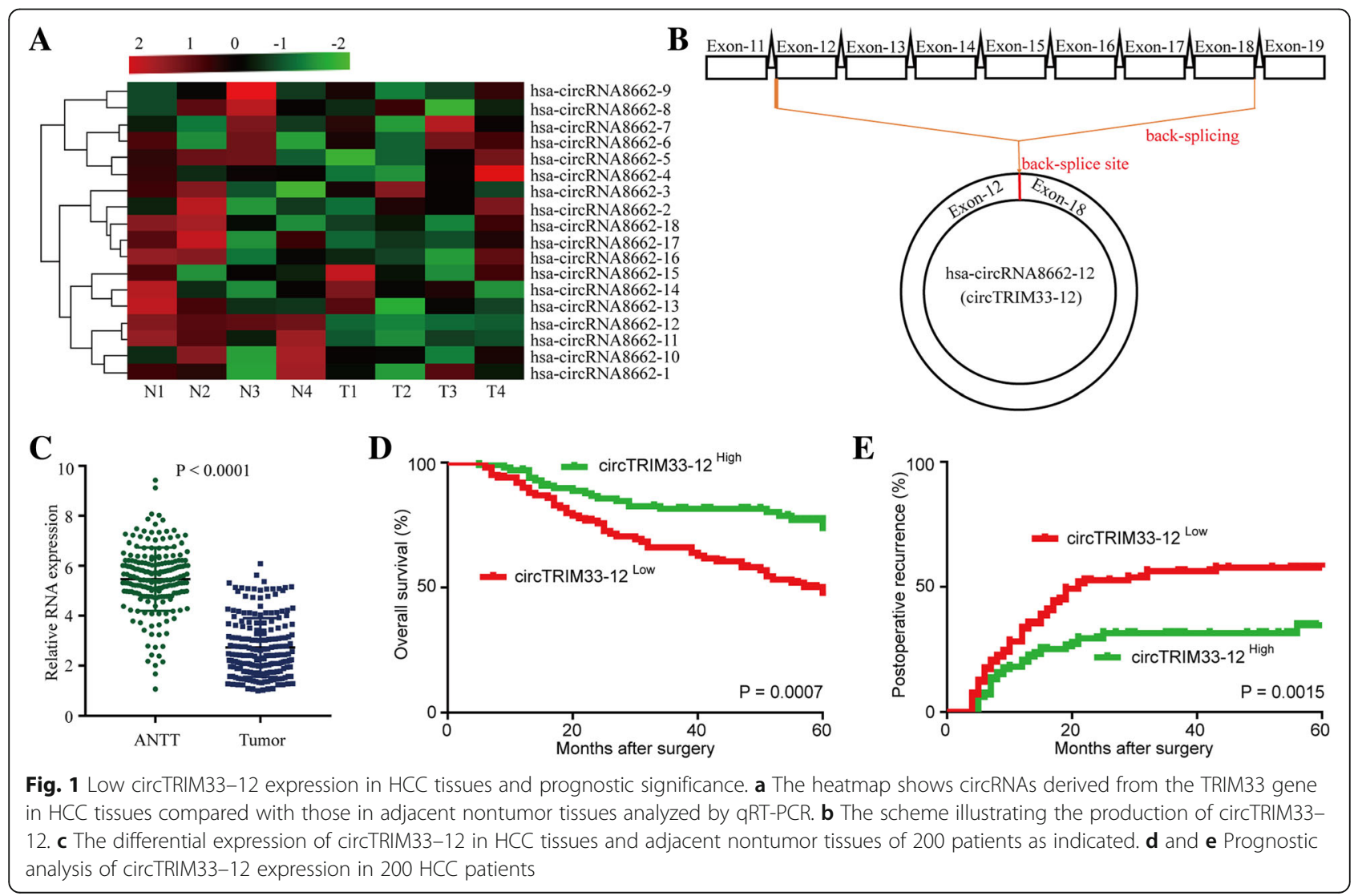


1c). The results showed that circTRIM33-12 was significantly reduced in 58\% (116/200) of the HCC tissues examined compared to the levels of circTRIM33-12 in the matched adjacent tissues from the same patient. The median relative expression level of circTRIM33-12 from all $200 \mathrm{HCC}$ tissues was chosen as the cut-off point for separating tumors with the high expression of circTRIM33-12 and the low expression of circTRIM33-12. Next, we investigated the relationship between circTRIM33-12 expression and the clinicopathological characteristics in 200 HCC patients, as listed in Table 1. The results demonstrate that HCC patients with cirCTRIM33-12 ${ }^{\text {low }}$ had larger tumor sizes $(P=0.022)$, multiple tumors $(P=0.028)$, encapsulation invasion $(P=$ 0.007 ), elevated alphafetoprotein levels (AFP, $P=0.02$ ), and microvascular invasion $(P=0.002)$ compared with those in HCC patients with circTRIM33-12 $2^{\text {high }}$. KaplanMeier survival analysis revealed that patients with low circTRIM33-12 expression levels had a poor clinical outcome (Fig. 1d and e). Multivariate analysis identified circTRIM33-12 expression as an independent predictor for overall survival (OS) and postoperative recurrence (Tables 2 and 3). Together, these data suggest that decreased circTRIM33-12 expression in HCC cells is correlated with the poor prognosis of patients and indicate that reduced circTRIM33-12 expression is likely involved in the progression of HCC.

\section{circTRIM33-12 inhibits the proliferation and invasion of HCC cells}

Next, the biological functions of circTRIM33-12 in HCC progression were investigated. To choose the most suitable HCC cell lines used for the knockdown or overexpression of circTRIM33-12, we detected the expression of circTRIM33-12 in seven HCC cell lines. SMMC-7721 and HCCLM3 cells showed a lower expression of circTRIM3312, and Huh 7, HepG2, and MHCC97L cells showed a higher expression of circTRIM33-12 (Additional file 6: Figure S1a). Therefore, Huh 7 and MHCC97L cells were used for the knockdown of circTRIM33-12; SMMC-7721 and HCCLM3 cells were used for the overexpression of circTRIM33-12. Using small interfering RNAs (siRNAs) targeting the back-splicing sequence, we effectively knocked down circTRIM33-12 in Huh 7 and MHCC97L cells (Additional file 6: Figure S1b). To convincingly show that circTRIM33-12 siRNA had no effects on other TRIM33 splicing products, qRT-PCR was performed to detect the TRIM33 mRNA expression. The expression of TRIM33 mRNA showed no significant change after the transfection of circTRIM33-12 siRNA, confirming the specificity of circTRIM33-12 silencing (Additional file 6: Figure S1c). Furthermore, using the plasmid vector, we succeeded in overexpressing circTRIM33-12 in SMMC-7721 and HCCL M3 cells and further obtained stable circTRIM33-12-
Table 1 Correlation between circTRIM33-12 and clinicopathological characteristics in 200 HCCs

\begin{tabular}{|c|c|c|c|}
\hline \multirow[t]{2}{*}{ Variable } & \multicolumn{2}{|c|}{ circTRIM33-12 } & \multirow{2}{*}{$\begin{array}{l}P \\
\text { value }\end{array}$} \\
\hline & Low & High & \\
\hline \multicolumn{4}{|l|}{ Age (years) } \\
\hline$\leq 50$ & 44 & 46 & 1.000 \\
\hline$>50$ & 56 & 55 & \\
\hline \multicolumn{4}{|l|}{ Sex } \\
\hline Female & 13 & 17 & 0.553 \\
\hline Male & 87 & 83 & \\
\hline \multicolumn{4}{|l|}{ HBsAg } \\
\hline Negative & 21 & 20 & 1.000 \\
\hline Positive & 79 & 80 & \\
\hline \multicolumn{4}{|l|}{ HCVAb } \\
\hline Negative & 3 & 4 & 1.000 \\
\hline Positive & 97 & 96 & \\
\hline \multicolumn{4}{|c|}{ Liver cirrhosis } \\
\hline No & 87 & 83 & 0.553 \\
\hline Yes & 13 & 17 & \\
\hline \multicolumn{4}{|c|}{ Serum AFP, ng/mL } \\
\hline$\leq 20$ & 30 & 47 & 0.02 \\
\hline$>20$ & 70 & 53 & \\
\hline \multicolumn{4}{|c|}{ Serum ALT, U/L } \\
\hline$\leq 75$ & 86 & 86 & 1.000 \\
\hline$>75$ & 14 & 14 & \\
\hline \multicolumn{4}{|c|}{ Tumor size (diameter, cm) } \\
\hline$\leq 5$ & 49 & 66 & 0.022 \\
\hline$>5$ & 51 & 34 & \\
\hline \multicolumn{4}{|c|}{ Encapsulation invasion } \\
\hline Absent & 38 & 58 & 0.007 \\
\hline Present & 62 & 42 & \\
\hline \multicolumn{4}{|c|}{ Vascular invasion } \\
\hline No & 51 & 73 & 0.002 \\
\hline Yes & 49 & 27 & \\
\hline \multicolumn{4}{|c|}{ Tumor number } \\
\hline Single & 75 & 88 & 0.028 \\
\hline Multiple & 25 & 12 & \\
\hline \multicolumn{4}{|l|}{ TNM } \\
\hline l/ II & 73 & 71 & 0.875 \\
\hline III/IV & 27 & 29 & \\
\hline
\end{tabular}


Table 2 Univariate and Multivariate Analyses of Factors Associated with Overall Survival

\begin{tabular}{|c|c|c|c|c|}
\hline \multirow[b]{3}{*}{ Factors } & \multicolumn{4}{|l|}{ OS } \\
\hline & \multirow[b]{2}{*}{ Univariate, $\mathrm{P}$} & \multicolumn{2}{|c|}{ Multivariate } & \multirow[b]{2}{*}{$P$ value } \\
\hline & & $\mathrm{HR}$ & $95 \% \mathrm{Cl}$ & \\
\hline Sex (female vs. male) & 0.106 & & & NA \\
\hline Age (years) ( $\leq 50$ vs. $>50$ ) & 0.118 & & & NA \\
\hline HBsAg (positive vs. negative) & 0.730 & & & NA \\
\hline HCVAb (positive vs. negative) & 0.860 & & & NA \\
\hline Liver cirrhosis (yes vs. no) & 0.598 & & & NA \\
\hline Serum AFP, ng/mL ( $\leq 20$ vs. $>20)$ & 0.424 & & & NA \\
\hline Serum ALT, U/L ( $\leq 75$ vs. > 75) & 0.627 & & & NA \\
\hline Tumor size (diameter, $\mathrm{cm})(>5 \mathrm{vs} \leq 5)$ & 0.004 & 1.172 & $1.066-2.750$ & 0.026 \\
\hline Tumor number (multiple vs. single) & 0.175 & & & NA \\
\hline TNM (III/IV vs I/II.) & 0.121 & & & NA \\
\hline CircTRIM33-12 expression (high vs. low) & 0.001 & 0.504 & $0.932-1.942$ & 0.007 \\
\hline
\end{tabular}

OS overall survival, NA not adopted, AFP alpha-fetoprotein, $H B s A g$ hepatitis B surface antigen, $95 \% C l$ 95\% confidence interval, $H R$ hazard ratio, Cox proportional hazards regression model

overexpressing SMMC-7721 and HCCLM3 clones which showed approximately four fold overexpression (Additional file 6: Figure S1d).

The CCK-8 assay, cell cycle assay, and transwell migration and invasion assay revealed a significant inhibition in the growth and metastasis of SMMC-7721 and HCCLM3 cells after overexpressing circTRIM33-12 compared with the growth and metastasis of the mock control (Fig. 2a-c). Moreover, the knockdown of circTRIM33-12 expression promoted the growth and metastasis of Huh 7 and MHCC97L cells (Additional file 6: Figure S2a-c). To further explore the effects of circTRIM33-12 in vivo, circTRIM 33-12-overexpressing SMMC-7721 cells and mock control cells were applied to establish orthotopic implanted intrahepatic HCC models. The size of the tumors was smaller in the circTRIM33-12-overexpressing group compared with the size of the tumors in the mock control after in situ growth for 4 weeks in SMMC-7721 xenografts (Fig. 2d). Consistent with these expectations, the metastatic nodules in the pulmonary tissues were reduced in the circTRIM33-12-overexpressing group compared with those in the mock control group in SMMC-7721 xenografts (Fig. 2e).

circTRIM33-12 may function as a sponge for miR-191 Given that circRNAs have been shown to act as miRNA sponges in cancer cells, we explored whether circTRIM3312 can bind to certain miRNAs in the progression of HCC.

Table 3 Univariate and Multivariate Analyses of Factors Associated with Cumulative Recurrence

\begin{tabular}{|c|c|c|c|c|}
\hline \multirow[b]{3}{*}{ Factors } & \multicolumn{4}{|c|}{ Cumulative Recurrence } \\
\hline & \multirow[b]{2}{*}{ Univariate, $\mathrm{P}$} & \multicolumn{2}{|c|}{ Multivariate } & \multirow[b]{2}{*}{$P$ value } \\
\hline & & $\mathrm{HR}$ & $95 \% \mathrm{Cl}$ & \\
\hline Sex (female vs. male) & 0.161 & & & NA \\
\hline Age (years) ( $\leq 50$ vs. $>50)$ & 0.184 & & & NA \\
\hline HBsAg (positive vs. negative) & 0.419 & & & NA \\
\hline HCVAb (positive vs. negative) & 0.708 & & & NA \\
\hline Liver cirrhosis (yes vs. no) & 0.489 & & & NA \\
\hline Serum AFP, ng/mL ( $\leq 20$ vs. $>20)$ & 0.182 & & & NA \\
\hline Serum ALT, U/L ( $\leq 75$ vs. $>75)$ & 0.622 & & & NA \\
\hline Tumor size (diameter, cm) (> 5 vs $\leq 5$ ) & $<0.001$ & 2.055 & $1.335-3.163$ & 0.001 \\
\hline Tumor number (multiple vs. single) & 0.131 & & & NA \\
\hline TNM (III/IV vs I/II.) & 0.099 & & & NA \\
\hline CircTRIM33-12 expression (high vs. low) & 0.001 & 0.534 & $0.344-0.830$ & 0.005 \\
\hline
\end{tabular}




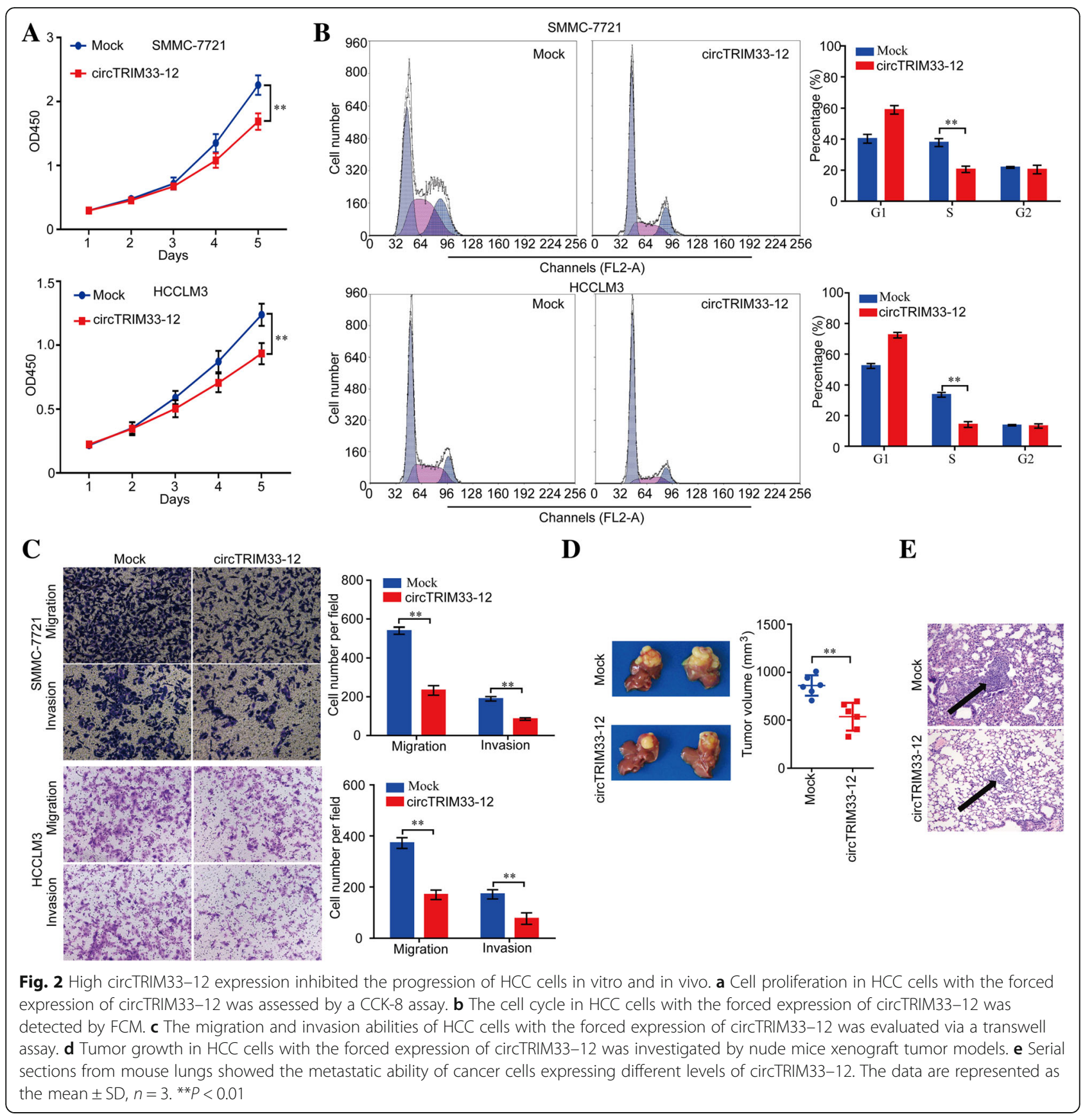

First, we conducted RIP with an antibody against AGO2 in Huh 7 cells. The results showed that circTRIM33-12, but not cANRIL (a circular RNA reported not to bind to AGO2), was significantly enriched by the AGO2 antibody (Fig. 3a), suggesting that circTRIM33-12 may act as a binding platform for AGO2 and miRNAs. Next, we used the StarBase v2.0 target prediction tool to find 11 potential miRNAs that could bind to circTRIM33-12. According to recent studies, three these of miRNAs act as oncogenes, including miR-23a-3p, miR-191, and miR-224-5p. To further identify the miRNAs miR-23a-3p, miR-191 and miR-224-
$5 \mathrm{p}$ that bind to circTRIM33-12, we constructed a circTRIM33-12 expression plasmid vector containing a luciferase gene and transfected the plasmid vector into Huh 7 cells. After transfection, we observed that 3 miRNAs, particularly miR-191, showed reduced levels of luciferase activity (Fig. $3 \mathrm{~b}$ and c). Furthermore, the results of a pull-down assay using biotin-labeled miR-191 mimics showed obvious enrichment of circTRIM33-12 compared with that of the negative control (NC) (Fig. 3d). However, circTRIM33-12 did not show significant changes after increased or reduced miR-191 mimic expression in HCC Huh 7 cells (Fig. 3e), 


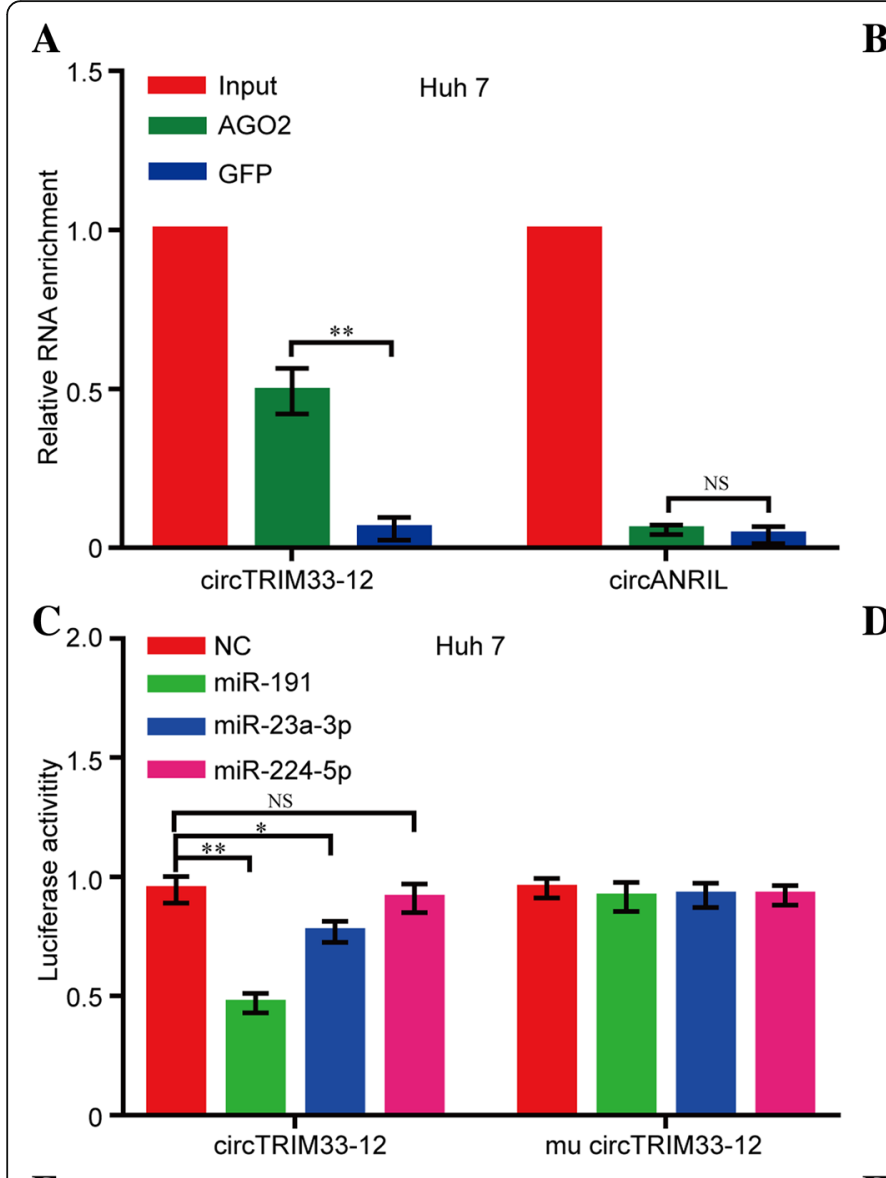

B

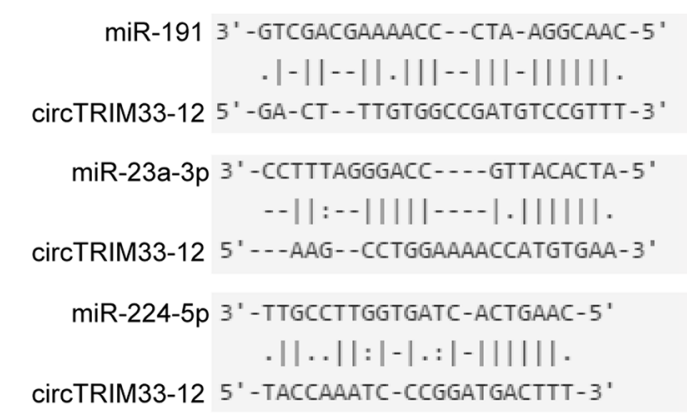

D
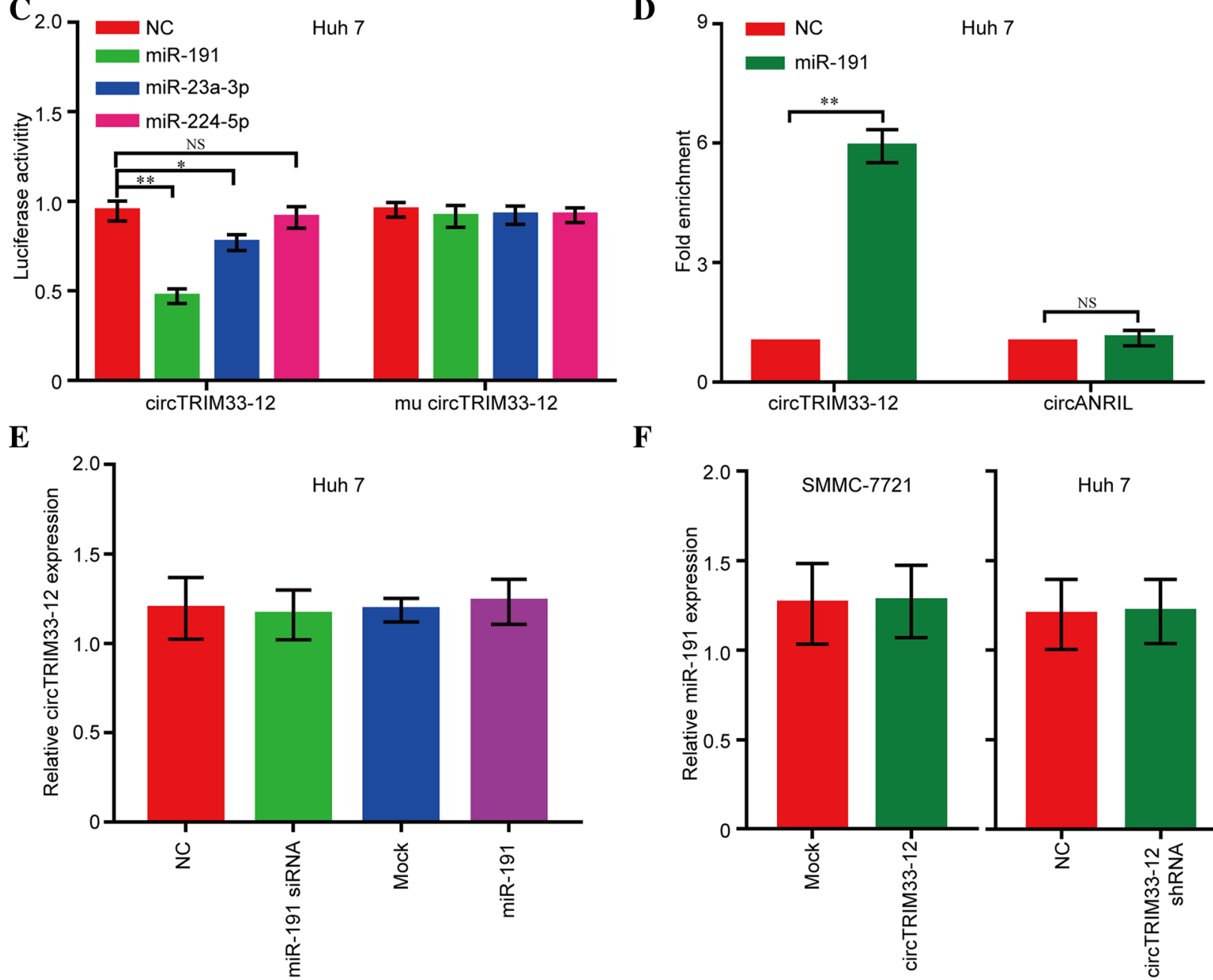

F

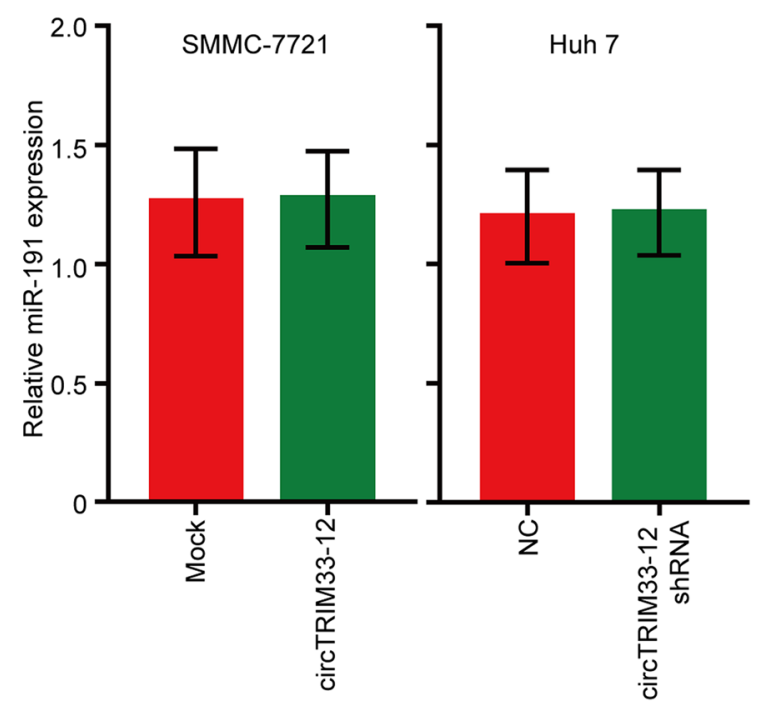

Fig. 3 circTRIM33-12 binds miR-191 in HCC cells. a RIP experiments were performed using an antibody against AGO2 on extracts from Huh 7 cells. b A schematic drawing showing the putative binding sites of miR-23a-3p, miR-191, and miR-224-5p with respect to circTRIM33-12. c The luciferase activity of luc-circTRIM33-12 or mutant luc-circTRIM33-12 in Huh 7 cells after cotransfection with miR-23a-3p, miR-191, or miR-224-5p. d circRIP was performed in Huh 7 cells using biotin-labeled miR-191 mimics and a negative control (NC). The enrichment of circTRIM33-12 was detected by qRT-PCR and was normalized to that of the NC. e The expression of circTRIM33-12 in Huh 7 cells after transfection with miR-191 or miR-191 siRNA. $\mathbf{f}$ The expression of circ miR-191 in SMMC-7721 and Huh 7 cells after transfection with circTRIM33-12 or circTRIM33-12 shRNA. The data are represented as the mean $\pm \mathrm{SD}, n=3 .{ }^{*} P<0.05 ;{ }^{* *} P<0.01 ; \mathrm{NS}$, no significant 
and neither miR-191 showed significant changes after the forced expression or knockdown of circTRIM33-12 expression in HCC SMMC-7721 or Huh 7 cells (Fig. 3f). These findings suggest that circTRIM33-12 and miR-191 may not be degraded by each other. All these experiments suggest that circTRIM33-12 may function as a sponge for miR-191.

\section{circTRIM33-12 upregulates TET1 expression via sponging miR-191}

Previous studies have reported that several genes are directly targeted by miR-191 in human cancers including TET1, TIMP3, SATB1, and DIECR1 [27-29]. However, we did not detect significant changes in the expression levels of these mRNAs in Huh 7 cells transfected with a miR-191 mimic or in SMMC-7721 cells transfected with a miR-191 siRNA. Interestingly, we also did not detect significant changes in the expression levels of these proteins in miR-191-overexpressing/knockdown cells except for the expression of the TET1 protein (Additional file 6: Figure S3a-d). To verify whether the 3' UTR of TET1 mRNA was a target of miR-191 in HCC cells, a luciferase reporter gene assay was used. The wild-type (wt) 3' UTR sequence or mutant (mu) 3' UTR sequence of TET1 was cloned into a luciferase reporter vector. The luciferase activity was significantly inhibited by the miR-191 mimics in wt 3' UTR sequence-transfected Huh 7 cells. Conversely, the luciferase activity was not inhibited by the miR-191 mimics in mu 3' UTR sequence-transfected Huh 7 cells (Additional file 6: Figure S3e and f). These findings indicate a direct interaction between miR-191 and TET1 mRNA in HCC cells. To further confirm the effects of circTRIM3312 on TET1 expression, SMMC-7721 cells were transfected with the circTRIM33-12 plasmid, and the TET1 mRNA and protein levels were detected using qRT-PCR and western blotting. The results showed that the ectopic expression of circTRIM33-12 significantly increased the TET1 protein levels but not mRNA levels in SMMC-7721 cells (Fig. 4a and b). Conversely, the knockdown of circTRIM33-12 expression significantly reduced the TET1 protein levels but not mRNA levels in Huh 7 cells (Fig. 4c and d). A luciferase activity assay demonstrated that the knockdown of circTRIM33-12 could effectively reduce the luciferase activity of the wt but not the mu 3' UTR of TET1, and the inhibition of luciferase activity caused by circTRIM33-12 knockdown could be restored by miR191 inhibition in Huh 7 cells (Fig. 4e and f). Moreover, the forced expression of circTRIM33-12 could effectively increase the luciferase activity of the wt but not the mu TET1 3' UTR in SMMC-7721 cells (Fig. 4f). Finally, we determined the expression of TET1 in 200 HCC patient tissues using $\mathrm{qRT}-\mathrm{PCR}$ and IHC. A positive relationship between circTRIM33-12 and TET1 was found in HCC patients (Fig. $4 \mathrm{~g}$ and $\mathrm{h}$ ).

\section{circTRIM33-12 and TET1 are responsible for regulating} $5 \mathrm{hmC}$ expression

To test whether the altered circTRIM33-12 or TET1 expression could regulate the DNA methylation levels in HCC cells, circTRIM33-12 expression in HCC cells was modified by shRNA interference and cDNA transfection (Additional file 6: Figure S4a-c). We first compared the $5 \mathrm{mC}$ and $5 \mathrm{hmC}$ levels in mock- and circTRIM33-12- or TET1-overexpressing SMMC-7721 and HCCLM3 cells. We found that the levels of $5 \mathrm{hmC}$ were significantly increased in the circTRIM33-12- or TET1-overexpressing cells, as shown by the IF assay (Fig. $5 \mathrm{a}$ and b). Conversely, the levels of $5 \mathrm{hmC}$ were significantly reduced in circTRIM33-12 or TET1-knockdown Huh 7 and MHCC97L cells as shown by the IF assay (Additional file 6: Figure S5ab). Interestingly, growth curves demonstrated that TET1 significantly inhibited cell proliferation compared with the cell proliferation of the mock groups (Fig. 5c). Moreover, in the cell cycle assay, TET1 reduced the cell number in the $\mathrm{S}$ phase compared with those in the mock groups (Fig. 5d). In vitro migration and invasion assays were performed to explore whether TET1 inhibited the migration and invasive capacity of tumor cells. In addition to circTRIM33-12, TET1 significantly inhibited SMMC-7721 cell migration and invasion compared with those of the mock group (Fig. 5e). Moreover, the capacity of proliferation, migration and invasion were significantly inhibited in TET1 knockdown Huh 7 and MHCC97L cells as shown by the CCK-8, cell cycle, and transwell assays (Additional file 6: Figure S5c-e). These data convincingly demonstrate that circTRIM33-12 suppresses HCC progression as a sponge of miR-191 to inhibit the miR-191 oncogenic effects through the circTRIM33-12/miR-191/TET1 axis.

\section{TET1 negatively regulates oncogene expression and is related to immune evasion}

To further gain a deeper mechanistic understanding of the circTRIM33-12/ miR-191/ TET1 axis, potential transcriptomic regulation by TET1 was explored in TET1 knockdown and negative control Huh 7 cells using RNA sequencing analysis. $5 \mathrm{mC}$ is an important epigenetic marker associated with transcription repression. We next sought to investigate whether TET1 knockdown transcriptionally inhibits tumor suppressor gene expression in HCC cells. The RNA-seq results showed that TET1 knockdown downregulated several tumor suppressor genes that participated in tumor progression, such as WWC family member 3 (WWC3), tumor protein p53 inducible nuclear protein 1 (TP53INP1), UL16 binding protein 1 (ULBP1) and lysine demethylase 7A (JHDM1D) (Additional file 7: Table S5). To investigate whether circTRIM33-12 exerts its biological functions through the miR-191/TET1 axis, we examined the expression of WWC3, TP53INP1, ULBP1 and JHDM1D in HCC cells with circTRIM33-12 or TET1 knockdown 


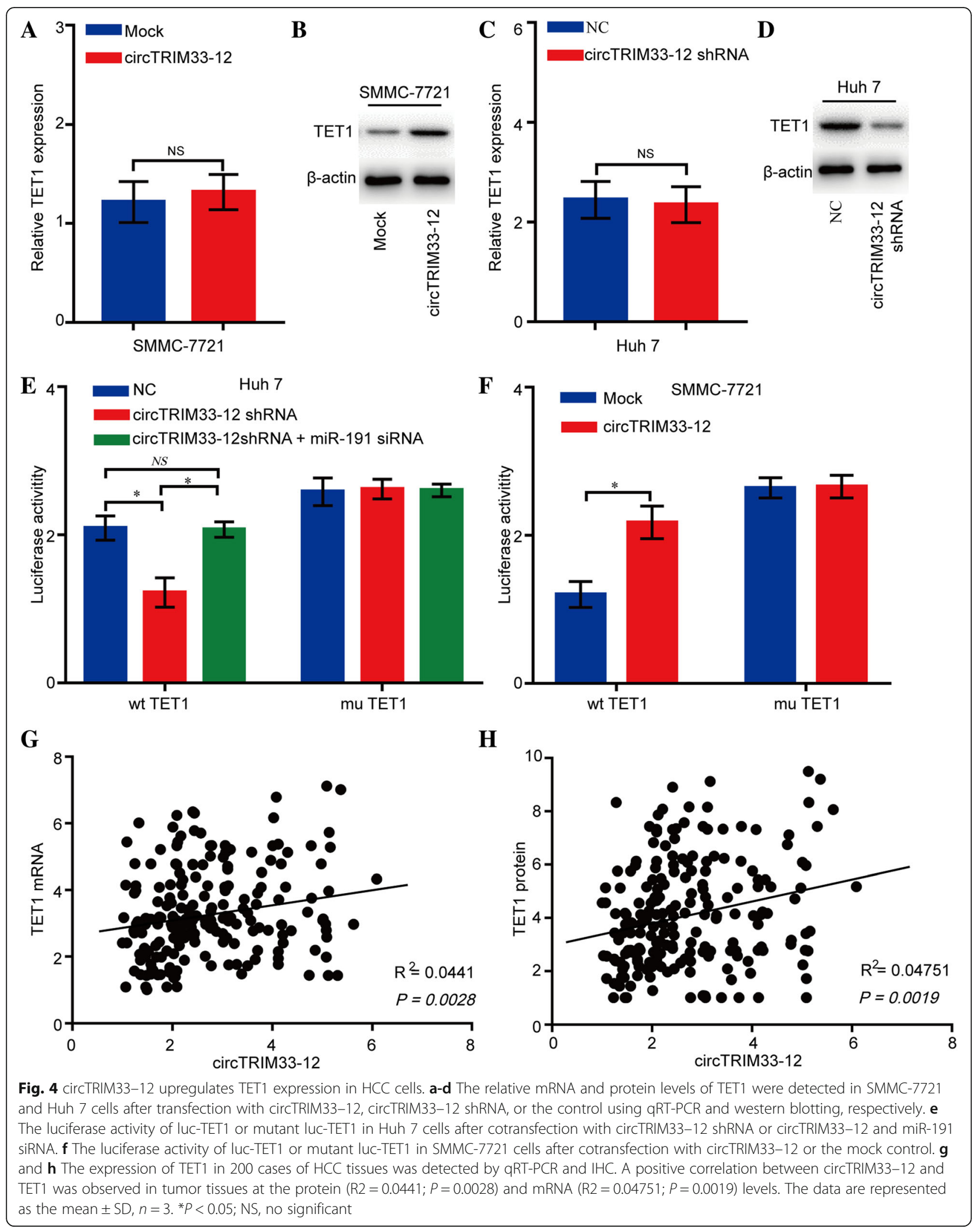




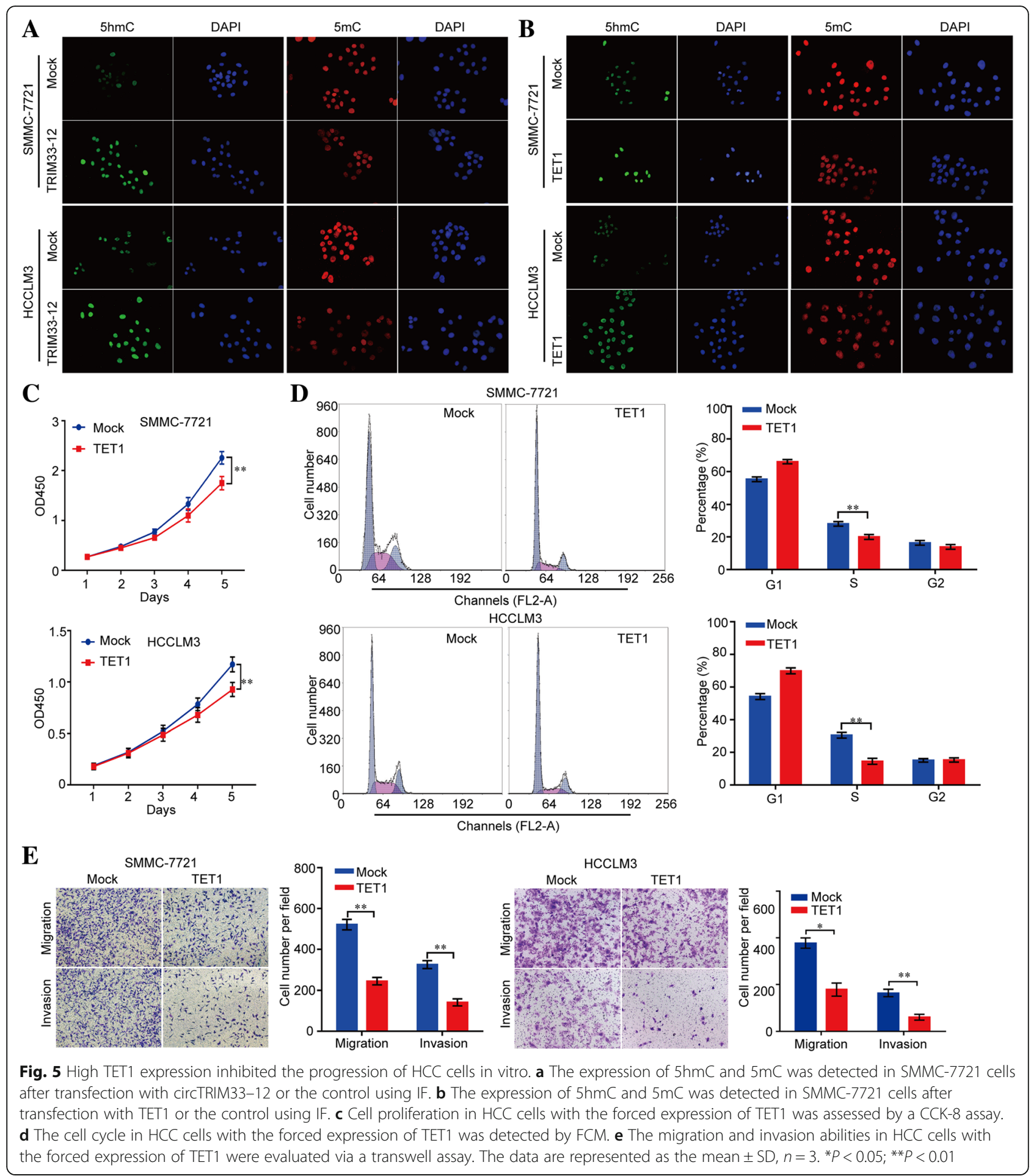

and overexpression. Both the silencing of circTRIM33-12 or TET1 significantly reduced the mRNA and protein expression levels of WWC3, TP53INP1, ULBP1 and JHDM1D in HCC Huh 7 cells (Fig. 6a; Additional file 6: Figure S6a). In addition, the overexpression of circTRIM33-12 or TET1 could increase the mRNA and protein expression levels of WWC3, TP53INP1, ULBP1 and JHDM1D in HCC SMMC-
7721 cells (Fig. 6b; Additional file 6: Figure S6b). To verify whether the 3' UTR of the WWC3, TP53INP1, ULBP1 and JHDM1D mRNAs were targets of miR-191 in HCC cells, a luciferase reporter gene assay was used. The 3' UTR sequences of WWC3, TP53INP1, ULBP1 and JHDM1D were cloned into a luciferase reporter vector. The luciferase activity was not inhibited by the miR-191 mimics in 3' UTR 

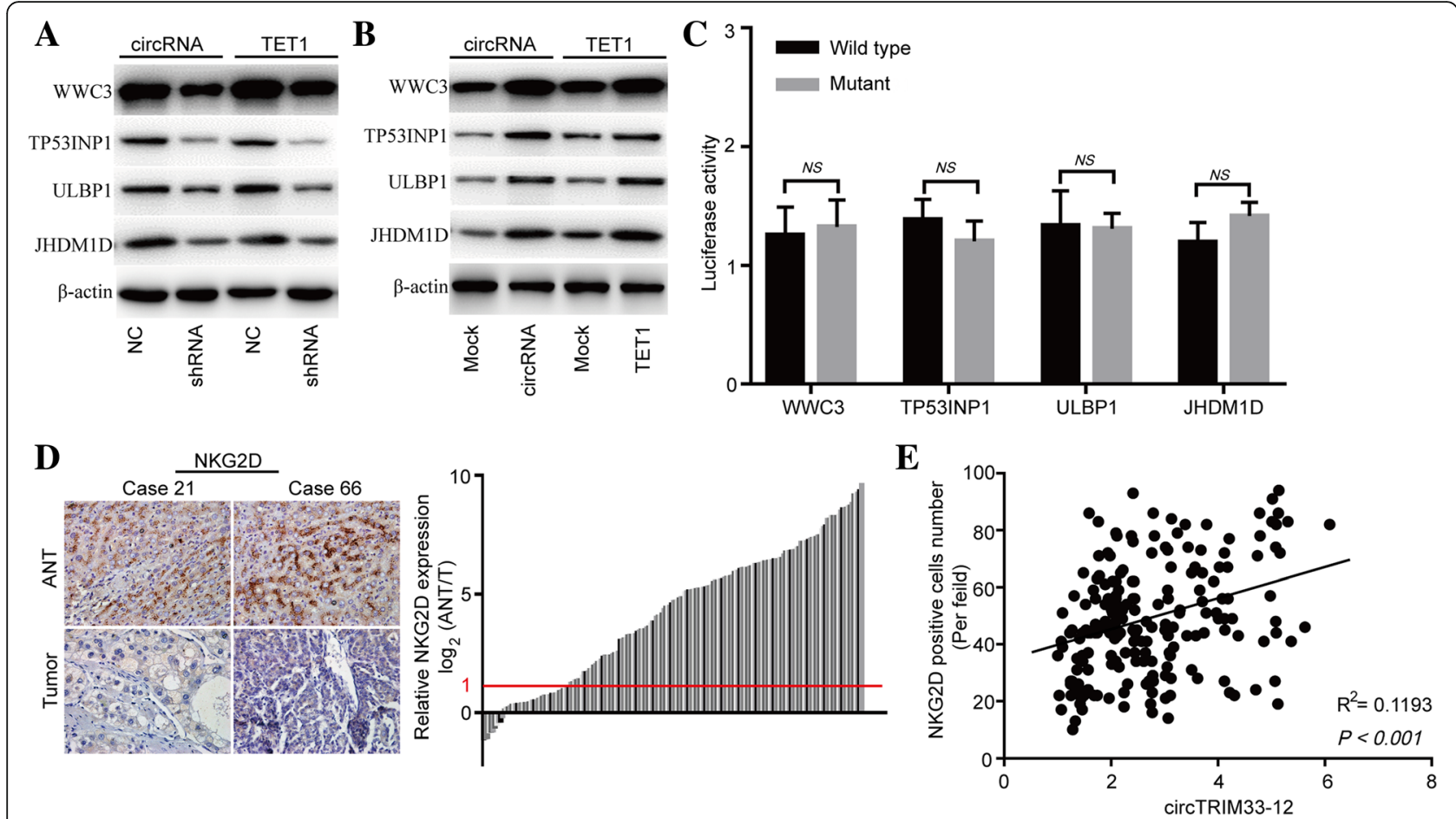

Fig. 6 circTRIM33-12 and TET1 regulate the expression of several same genes in HCC cells. a The protein levels of WWC3, TP53INP1, ULBP1 and JHDM1D were detected in SMMC-7721 cells after transfection with circTRIM33-12, TET1, or the control using western blotting. b The protein levels of WWC3, TP53INP1, ULBP1 and JHDM1D were detected in Huh 7 cells after transfection with circTRIM33-12 shRNA, TET1 shRNA, or the control using western blotting. c The luciferase activity of luc-WWC3/TP53INP1/ULBP1/JHDM1D or mutant luc-WWC3/TP53INP1/ULBP1/JHDM1D in Huh 7 cells after cotransfection with miR-191. d Representative HCC cases in the tissue microarray were analyzed by immunohistochemical staining for NKG2D. e A positive correlation between circTRIM33-12 and the number of NKG2D-positive cells was observed in $H C C$ tissues $\left(R^{2}=\right.$ $0.1193 ; P<0.001)$. The data are represented as the mean $\pm S D, n=3$. NS, no significant

sequence-transfected Huh 7 cells (Fig. 6c). These findings indicate that WWC3, TP53INP1, ULBP1 and JHDM1D mRNAs are not targets of miR-191 in HCC cells.

The activating receptor natural-killer group 2 member D (NKG2D) and its ligands play vital roles in NK cell-, $\gamma \delta+T$ cell- and CD8+ T cell-mediated immune responses to cancers [30,31]. NKG2D recognizes eight different ligands in humans, including ULBP1 [32]. One of the important mechanisms that prevents cancer progression is immune surveillance against cancer cells, in which NK cells and CD8+ T cells play a crucial role $[33,34]$. To further explore the relationship between circTRIM33-12 and immune evasion, we examined the expression of NKG2D in tissues from 200 cases of HCC and the matched nontumor tissues. The number of NKG2D-positive cells in HCC tissues were significantly reduced compared with that in the adjacent nontumor tissues (156/200; 2 fold) (Fig. 6d). A scatter plot analysis revealed a positive correlation between circTRIM33-12 expression and NKG2D-positive cell number in HCC tissues $\left(R^{2}=0.1193 ; P<0.001\right.$; Fig. 6e). These results suggest that circTRIM33-12 may exert its antitumor effects by protecting TET1 via sponging miR-191.
Silencing of miR-191 reversed the knockdown of circTRIM33-12 and induced the progression of HCC cells We next explored whether the silencing of miR-191 affected circTRIM33-12 knockdown HCC cell proliferation, migration, and invasion. The silencing efficiency was detected by qRT-PCR (Fig. 7 a). As expected, the silencing of miR-191 upregulated TET1 protein expression but not mRNA expression in both Huh 7 and MHCC97L cell lines with circTRIM33-12 knockdown (Fig. $7 \mathrm{~b}$ and c). Furthermore, in the CCK- 8 and transwell assays, the silencing of miR-191 significantly inhibited the proliferation, migration, and invasion abilities of Huh 7 and MHCC97L cell lines with reduced levels of circTRIM33-12 expression (Fig. 7d-g). Collectively, these results suggest that circTRIM33-12 suppresses HCC cell progression through the circTRIM33-12/miR191/TET1 axis.

\section{Discussion}

In this study, we first discovered that circTRIM33-12 is frequently downregulated in $\mathrm{HCC}$, and its expression significantly correlated with better clinicopathologic 


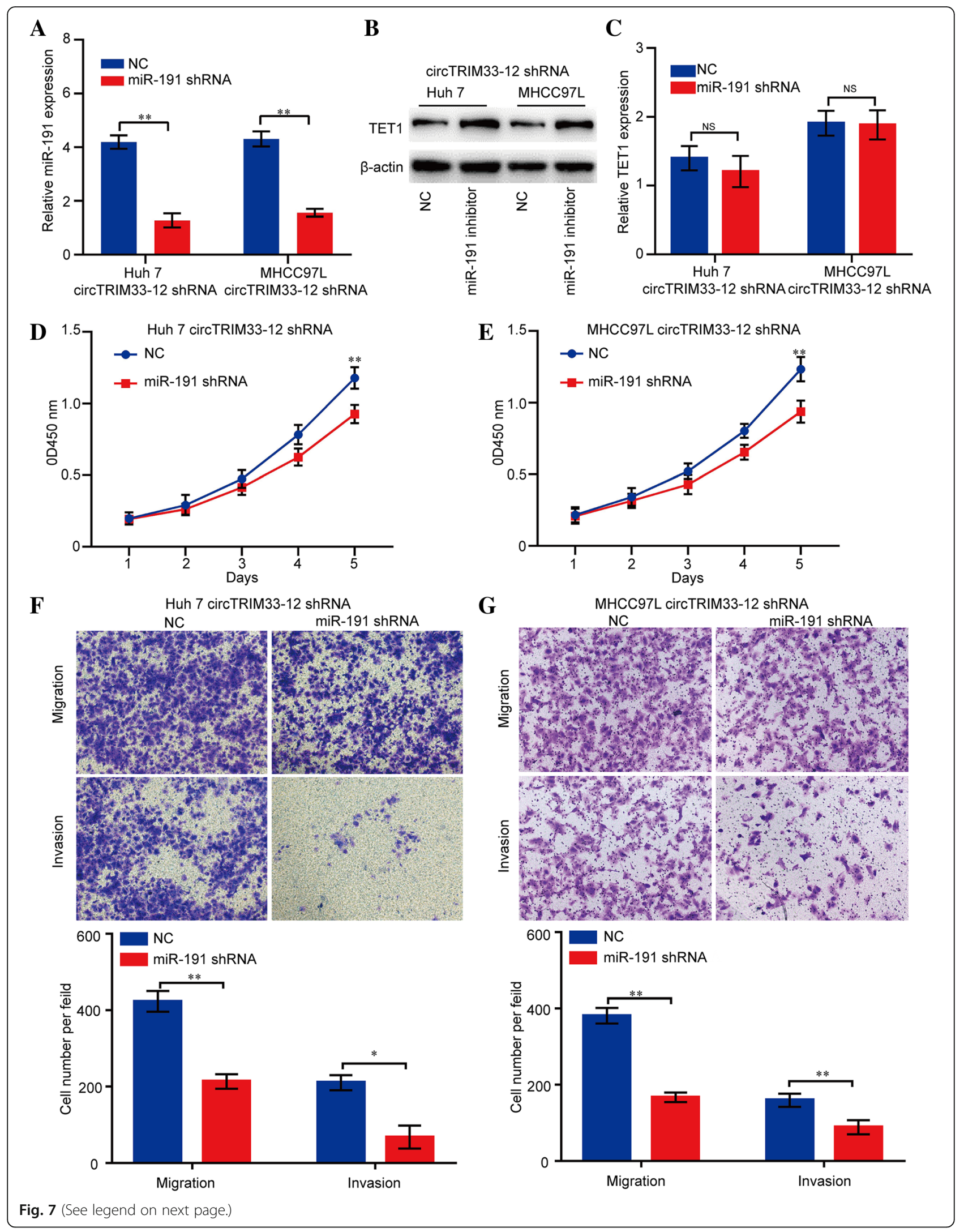


(See figure on previous page.)

Fig. 7 Silencing of miR-191 impairs the cell proliferation, migration and invasion abilities of HCC cells with reduced circTRIM33-12 expression. a miR-191 expression in HCC cells was modified by shRNA interference. $\mathbf{b}, \mathbf{c}$ Reduced miR-191 expression enhanced TET1 protein expression but not TET1 mRNA in HCC cells. $\mathbf{d}$, e The cell proliferation in HCC cells with the reduced expression of miR-191 was assessed by a CCK-8 assay. $\mathbf{f}, \mathbf{g}$ The migration and invasion abilities in HCC cells with the reduced expression of miR-191 were evaluated via a transwell assay. The data are represented as the mean $\pm S D, n=3$. ${ }^{*} P<0.05$; ${ }^{* *} P<0.01$; NS, no significant

characteristics. Second, our data showed that the low expression of circTRIM33-12 correlated with poor patient prognosis, indicating its applicability as a promising prognostic biomarker in HCC. Third, we demonstrated that the overexpression of circTRIM33-12 reversed the oncogenic function of miR-191 and thus inhibited the progression of HCC. Fourth, we revealed that circTRIM 33-12 acts as a ceRNA and regulates TET1-induced DNA demethylation by competing for miR-191. Considering the vital role of TET1-induced DNA demethylation in the progression of cancers, our results unraveled the therapeutic importance of circTRIM33-12 in HCC for the first time.

Currently, the roles of circRNAs in carcinogenesis and cancer progression have attracted much attention; however, the biological functions and molecular mechanisms for circRNA function in these pathological processes have not been elucidated clearly. Recently, several studies have discovered that circRNA works as a miRNA sponge to protect mRNAs from miRNA attack $[14,35]$. For example, circMTO1 was reported to be a tumor-related molecule in HCC cells that functions by absorbing miR-9 [14]. Circular RNA cSMARC A5 functions as a sponge of miR-17-3p and miR-181b$5 \mathrm{p}$ to suppress the proliferation and migration of $\mathrm{HCC}$ cells [12]. circNT5E participates in glioblastoma tumorigenesis by interacting with miR-422a [35]. To investigate the expression and function of circRNAs derived from TRIM33 in HCC pathogenesis, we performed qRT-PCR to detect the expression levels of TRIM33-related circRNAs in 4 pairs of HCC and adjacent nontumor tissues. The results showed that the expression of circTRIM33-12 was reduced in all 4 HCC tissues. Herein, circTRIM33-12 has been shown to target miR-191 using bioinformatics tools. Intriguingly, the ectopic expression of miR-191 reduced the luciferase activity of the wt circTRIM33-12 reporter. However, there was no significant difference in circTRIM 33-12 expression upon forced miR-191 expression. Furthermore, endogenous circTRIM33-12 and miR191 were pulled down by a special AGO2 antibody. Taken together, all of the data suggest that miR-191 recognizes and binds to circTRIM33-12 without promoting the degradation of circTRIM33-12. miR-191 is an oncogene and is involved in cancer progression, including HCC [36, 37]. Because forced miR-191 expression promotes $\mathrm{HCC}$ cell proliferation, migration and invasion $[36,38]$, we reasoned that miR-191 may participate in the regulation of the circTRIM33-12-induced inhibition of proliferation, migration and invasion in HCC cells. TET1 is a methylcytosine dioxygenase that participates in epigenetic regulation [39]. TET1 protein can lead to DNA demethylation by oxidizing $5 \mathrm{mC}$ to $5 \mathrm{hmC}$, 5 -formylcytosine $(5 \mathrm{fC})$, and 5-carboxylcytosine $(5 \mathrm{caC})$ [40]. A previous study provided evidence that TET1 exerts its anti-tumor functions in cancer cells by several mechanisms, including suppressing $W n t / \beta$-catenin signaling via the demethylation of Wnt antagonists and promoting tumor suppressor gene expression by demethylating a CpG site within the tumor suppressor gene promoter [41-43]. However, the molecular mechanism of the induction of TET1 and its role in tumor progression are still obscure. Here, we found that cirCTRIM33-12 abolishes the suppression of TET1 mediated by miR-191, and the knockdown of TET1 contributes to the promotion of proliferation, migration and invasion. Therefore, we assumed that circTRIM33-12 could upregulate TET1 expression by competitively sponging miR-191, thereby inhibiting HCC progression. In addition, circTRIM33-12 facilitated tumor suppressor gene expression in HCC cells, such as WWC3, TP53INP1, ULBP1 and JHDM1D, via sponging the miR-191-induced upregulation of TET1 in HCC cells. Notably, the combination of circTRIM33-12 and miR-191/TET1 could be a potential prognostic biomarker panel of HCC. All of the above data suggest that the circTRIM33-12/miR-191/TET1 axis plays a critical role in HCC.

\section{Conclusions}

In summary, our findings report that circTRIM33-12 expression is significantly reduced in HCC tissues and that low levels of circTRIM33-12 expression correlate with a poor prognosis for HCC patients. Functionally and mechanistically, circTRIM33-12 inhibits HCC proliferation, metastasis and immune evasion by sponging miR-191 and upregulating TET1 expression, indicating its tumor suppressive role in HCC progression. Furthermore, the in vivo experiments and clinical characteristics of circTRIM33-12 indicate its potential in HCC-targeted therapy. Taken together, our data offer a novel therapeutic target, circTRIM33-12, to broaden the treatment options for HCC. 


\section{Additional files}

\section{Additional file 1: Supplementary Materials and Methods (DOC 34} kb)

Additional file 2: Table S1. Sequence of primers for qRT-PCR. (DOCX 17 $\mathrm{kb})$

Additional file 3: Table S2. Antibody for western blotting immunofluorescence, RIP, and immunohistochemistry. (DOCX 17 kb)

Additional file 4: Table S3. Target sequences of TET1 shRNA. (DOCX $15 \mathrm{~kb})$

Additional file 5: Table S4. circTRIM33-12 circRIP probe sequence. (DOCX 16 kb)

Additional file 6: Figure S1. circTRIM33-12 expression in HCC cells. Figure S2. circTRIM33-12 regulated the progression of HCC cells in vitro. Figure S3. TET1 binds to miR-191 in HCC cells. Figure S4. Forced or reduced TET1 expression in HCC cells. Figure S5. TET1 regulated the progression of HCC cells in vitro. Figure S6. circTRIM33-12 and TET1 regulate the expression of several same genes in HCC cells. (DOCX $3576 \mathrm{~kb}$ )

Additional file 7: Table S5. The significant reduced genes in TET1 knockdown cells. (XLSX 12 kb)

\section{Abbreviations}

5caC: 5-carboxylcytosine; 5fC: 5-formylcytosine;

5hmC: hydroxymethylcytosine; 5mC: 5-methylcytosine; AFP: Alphafetoprotein; AGO2: Argonaute 2; ANT: Adjacent normal tissue; circRNA: Circular RNA; HCC: Hepatocellular carcinoma; JHDM1D: Lysine demethylase 7A; NKG2D: Natural-killer group 2 member D; OS: Overall survival; qRTPCR: quantitative real-time polymerase chain reaction; RFS: Recurrence-free survival; RIP: RNA immunoprecipitation; shRNA: small hairpin RNA; siRNAs: small interfering RNAs; TET: Ten eleven translocation; TP53INP1: Tumor protein p53 inducible nuclear protein 1; TRIM33: Tripartite motif containing 33; ULBP1: UL16 binding protein 1; WWC3: WWC family member 3

\section{Acknowledgements}

Not applicable.

\section{Funding}

This study was funded by the National Natural Science Foundation of China (81502526, 81702861, 81702868), and the Outstanding Clinical Discipline Project of Shanghai Pudong (PWYgy2018-02).

\section{Availability of data and materials}

All data generated or analyzed during this study are included either in this article or in the supplementary Materials and Methods, Tables, Figures and Figure Legends files.

\section{Authors' contributions}

JF, GMS, and AWK conceived and designed the experiments; PFZ, CYW, XYH, $J C L$, and RP performed the experiments; $C Z$ and $C G$ analyzed the data; JBC, DMG, PFZ and AWK wrote the paper. All authors read and approved the final manuscript.

\section{Ethics approval and consent to participate}

Ethical approval was obtained from the Zhongshan Hospital Research Ethics Committee, and written informed consent was obtained from each patient.

\section{Consent for publication}

Not applicable.

\section{Competing interests}

The authors declare that they have no competing interests.

\section{Publisher's Note}

Springer Nature remains neutral with regard to jurisdictional claims in published maps and institutional affiliations.

\section{Author details}

'Liver Cancer Institute, Zhongshan Hospital, Key Laboratory of Carcinogenesis and Cancer Invasion, Fudan University, Ministry of Education, 180 Fenglin Road, Shanghai 200032, People's Republic of China. ${ }^{2}$ Cancer Center, Institutes of Biomedical Sciences, Fudan University, Shanghai 200031, People's Republic of China.

Received: 27 February 2019 Accepted: 15 May 2019

Published online: 01 June 2019

\section{References}

1. Llovet JM, Burroughs A, Bruix J. Hepatocellular carcinoma. Lancet. 2003; 362:1907-17.

2. Li Y, Tang Y, Ye L, Liu B, Liu K, Chen J, Xue Q. Establishment of a hepatocellular carcinoma cell line with unique metastatic characteristics through in vivo selection and screening for metastasis-related genes through cDNA microarray. J Cancer Res Clin Oncol. 2003;129:43-51.

3. Zhang Y, Li Q, Chen H. DNA methylation and histone modifications of Wnt genes by genistein during colon cancer development. Carcinogenesis. 2013; 34:1756-63.

4. Tan S, Gou Q, Pu W, Guo C, Yang Y, Wu K, Liu Y, Liu L, Wei YQ, Peng $Y$. Circular RNA F-circEA produced from EML4-ALK fusion gene as a novel liquid biopsy biomarker for non-small cell lung cancer. Cell Res. 2018:28:693-5.

5. Qiu M, Xia W, Chen R, Wang S, Xu Y, Ma Z, Xu W, Zhang E, Wang J, Fang T, et al. The circular RNA circPRKCl promotes tumor growth in lung adenocarcinoma. Cancer Res. 2018;78:2839-51.

6. Jeck WR, Sharpless NE. Detecting and characterizing circular RNAs. Nat Biotechnol. 2014:32:453-61.

7. Memczak S, Jens M, Elefsinioti A, Torti F, Krueger J, Rybak A, Maier L, Mackowiak SD, Gregersen LH, Munschauer M, et al. Circular RNAs are a large class of animal RNAs with regulatory potency. Nature. 2013;495:333-8.

8. Han B, Chao J, Yao H. Circular RNA and its mechanisms in disease: from the bench to the clinic. Pharmacol Ther. 2018;187:31-44.

9. Hansen TB, Jensen TI, Clausen BH, Bramsen JB, Finsen B, Damgaard CK, Kjems J. Natural RNA circles function as efficient microRNA sponges. Nature. 2013:495:384-8

10. Yang Y, Gao X, Zhang M, Yan S, Sun C, Xiao F, Huang N, Yang X, Zhao K, Zhou $\mathrm{H}$, et al. Novel role of FBXW7 circular RNA in repressing glioma tumorigenesis. J Natl Cancer Inst. 2018;110:304-15.

11. Li Z, Huang C, Bao C, Chen L, Lin M, Wang X, Zhong G, Yu B, Hu W, Dai L, et al. Exon-intron circular RNAs regulate transcription in the nucleus. Nat Struct Mol Biol. 2015;22:256-64.

12. Yu J, Xu QG, Wang ZG, Yang Y, Zhang L, Ma JZ, Sun SH, Yang F, Zhou WP. Circular RNA cSMARCA5 inhibits growth and metastasis in hepatocellular carcinoma. J Hepatol. 2018;68:1214-27.

13. Shi L, Yan $P$, Liang $Y$, Sun $Y$, Shen J, Zhou S, Lin H, Liang $X$, Cai X. Circular RNA expression is suppressed by androgen receptor (AR)-regulated adenosine deaminase that acts on RNA (ADAR1) in human hepatocellular carcinoma. Cell Death Dis. 2017:8:e3171.

14. Han D, Li J, Wang H, Su X, Hou J, Gu Y, Qian C, Lin Y, Liu X, Huang M, et al. Circular RNA circMTO1 acts as the sponge of microRNA-9 to suppress hepatocellular carcinoma progression. Hepatology. 2017;66:1151-64.

15. Ito S, D'Alessio AC, Taranova OV, Hong K, Sowers LC, Zhang Y. Role of Tet proteins in $5 \mathrm{mC}$ to $5 \mathrm{hmC}$ conversion, ES-cell self-renewal and inner cell mass specification. Nature. 2010:466:1129-33.

16. Ko M, Huang Y, Jankowska AM, Pape UJ, Tahiliani M, Bandukwala HS, An J, Lamperti ED, Koh KP, Ganetzky R, et al. Impaired hydroxylation of 5-methylcytosine in myeloid cancers with mutant TET2. Nature. 2010; 468:839-43.

17. Tahiliani M, Koh KP, Shen Y, Pastor WA, Bandukwala H, Brudno Y, Agarwal S, Iyer LM, Liu DR, Aravind L, Rao A. Conversion of 5-methylcytosine to 5hydroxymethylcytosine in mammalian DNA by MLL partner TET1. Science. 2009:324:930-5

18. Liu C, Liu L, Chen X, Shen J, Shan J, Xu Y, Yang Z, Wu L, Xia F, Bie P, et al. Decrease of 5-hydroxymethylcytosine is associated with progression of hepatocellular carcinoma through downregulation of TET1. PLoS One. 2013; 8:e62828.

19. Chuang KH, Whitney-Miller CL, Chu CY, Zhou Z, Dokus MK, Schmit S, Barry CT. MicroRNA-494 is a master epigenetic regulator of multiple invasion- 
suppressor microRNAs by targeting ten eleven translocation 1 in invasive human hepatocellular carcinoma tumors. Hepatology. 2015;62:466-80.

20. Ke AW, Shi GM, Zhou J, Huang XY, Shi YH, Ding ZB, Wang XY, Devbhandari RP, Fan J. CD151 amplifies signaling by integrin alpha6beta1 to PI3K and induces the epithelial-mesenchymal transition in HCC cells. Gastroenterology. 2011;140:1629-1641 e1615.

21. Huang XY, Ke AW, Shi GM, Zhang X, Zhang C, Shi YH, Wang XY, Ding ZB, Xiao YS, Yan J, et al. alphaB-crystallin complexes with 14-3-3zeta to induce epithelial-mesenchymal transition and resistance to sorafenib in hepatocellular carcinoma. Hepatology. 2013;57:2235-47.

22. Cai JB, Shi GM, Dong ZR, Ke AW, Ma HH, Gao Q, Shen ZZ, Huang XY, Chen $H, Y u$ DD, et al. Ubiquitin-specific protease 7 accelerates p14(ARF) degradation by deubiquitinating thyroid hormone receptor-interacting protein 12 and promotes hepatocellular carcinoma progression. Hepatology. 2015;61:1603-14.

23. Wang $H$, Zhou $M$, Shi B, Zhang $Q$, Jiang $H$, Sun $Y$, Liu J, Zhou K, Yao $\mathrm{M}, \mathrm{Gu}$ J, et al. Identification of an exon 4-deletion variant of epidermal growth factor receptor with increased metastasis-promoting capacity. Neoplasia. 2011:13:461-71.

24. Ding ZY, Jin GN, Wang W, Chen WX, Wu YH, Ai X, Chen L, Zhang WG, Liang $H F$, Laurence $A$, et al. Reduced expression of transcriptional intermediary factor 1 gamma promotes metastasis and indicates poor prognosis of hepatocellular carcinoma. Hepatology. 2014;60:1620-36.

25. Xue J, Chen Y, Wu Y, Wang Z, Zhou A, Zhang S, Lin K, Aldape K, Majumder S, Lu Z, Huang S. Tumour suppressor TRIM33 targets nuclear beta-catenin degradation. Nat Commun. 2015;6:6156.

26. Pommier RM, Gout J, Vincent DF, Alcaraz LB, Chuvin N, Arfi V, Martel S, Kaniewski B, Devailly G, Fourel G, et al. TIF1gamma suppresses tumor progression by regulating mitotic checkpoints and chromosomal stability. Cancer Res. 2015;75:4335-50

27. He Y, Cui Y, Wang W, Gu J, Guo S, Ma K, Luo X. Hypomethylation of the hsa-miR-191 locus causes high expression of hsa-mir-191 and promotes the epithelial-to-mesenchymal transition in hepatocellular carcinoma. Neoplasia. 2011;13:841-53.

28. Nagpal N, Ahmad HM, Molparia B, Kulshreshtha R. MicroRNA-191, an estrogen-responsive microRNA, functions as an oncogenic regulator in human breast cancer. Carcinogenesis. 2013;34:1889-99.

29. Zhang X, Wu M, Chong QY, Zhang W, Qian P, Yan H, Qian W, Zhang M, Lobie PE, Zhu T. Amplification of hsa-miR-191/425 locus promotes breast cancer proliferation and metastasis by targeting DICER1. Carcinogenesis. 2018:39:1506-16.

30. Bauer S, Groh V, Wu J, Steinle A, Phillips JH, Lanier LL, Spies T. Activation of NK cells and T cells by NKG2D, a receptor for stress-inducible MICA. Science. 1999;285:727-9.

31. Groschel C, Hubscher D, Nolte J, Monecke S, Sasse A, Elsner L, Paulus W, Trenkwalder C, Polic B, Mansouri A, et al. Efficient killing of murine pluripotent stem cells by natural killer (NK) cells requires activation by cytokines and partly depends on the activating NK receptor NKG2D. Front Immunol. 2017:8:870

32. Schmiedel D, Mandelboim O. NKG2D ligands-critical targets for Cancer immune escape and therapy. Front Immunol. 2018:9:2040.

33. Effros RB. Replicative senescence of CD8 T cells: potential effects on cancer immune surveillance and immunotherapy. Cancer Immunol Immunother. 2004:53:925-33.

34. Kamimura H, Yamagiwa S, Tsuchiya A, Takamura M, Matsuda Y, Ohkoshi S, Inoue M, Wakai T, Shirai Y, Nomoto M, Aoyagi Y. Reduced NKG2D ligand expression in hepatocellular carcinoma correlates with early recurrence. J Hepatol. 2012;56:381-8.

35. Wang R, Zhang S, Chen X, Li N, Li J, Jia R, Pan Y, Liang H. CircNT5E acts as a sponge of miR-422a to promote glioblastoma tumorigenesis. Cancer Res. 2018;78:4812-25.

36. Elyakim E, Sitbon E, Faerman A, Tabak S, Montia E, Belanis L, Dov A, Marcusson EG, Bennett CF, Chajut A, et al. Hsa-miR-191 is a candidate oncogene target for hepatocellular carcinoma therapy. Cancer Res. 2010;70: 8077-87.

37. Li H, Zhou ZQ, Yang ZR, Tong DN, Guan J, Shi BJ, Nie J, Ding XT, Li B, Zhou GW, Zhang ZY. MicroRNA-191 acts as a tumor promoter by modulating the TET1-p53 pathway in intrahepatic cholangiocarcinoma. Hepatology. 2017;66: $136-51$.
38. Huang D, Bi C, Zhao Q, Ding X, Bian C, Wang H, Wang T, Liu H. Knockdown long non-coding RNA ANRIL inhibits proliferation, migration and invasion of HepG2 cells by down-regulation of miR-191. BMC Cancer. 2018;18:919.

39. Zhang W, Xia W, Wang Q, Towers AJ, Chen J, Gao R, Zhang Y, Yen CA, Lee $A Y, L i Y$, et al. Isoform switch of TET1 regulates DNA demethylation and mouse development. Mol Cell. 2016;64:1062-73.

40. Pastor WA, Aravind L, Rao A. TETonic shift: biological roles of TET proteins in DNA demethylation and transcription. Nat Rev Mol Cell Biol. 2013;14:341-56.

41. Fan J, Zhang Y, Mu J, He X, Shao B, Zhou D, Peng W, Tang J, Jiang Y, Ren G, Xiang T. TET1 exerts its anti-tumor functions via demethylating DACT2 and SFRP2 to antagonize Wnt/beta-catenin signaling pathway in nasopharyngeal carcinoma cells. Clin Epigenetics. 2018;10:103.

42. Li BT, Yu C, Xu Y, Liu SB, Fan HY, Pan WW. TET1 inhibits cell proliferation by inducing RASSF5 expression. Oncotarget. 2017;8:86395-409.

43. Pei YF, Tao R, Li JF, Su LP, Yu BO, Wu XY, Yan M, Gu QL, Zhu ZG, Liu BY. TET1 inhibits gastric cancer growth and metastasis by PTEN demethylation and re-expression. Oncotarget. 2016;7:31322-35.

\section{Ready to submit your research? Choose BMC and benefit from:}

- fast, convenient online submission

- thorough peer review by experienced researchers in your field

- rapid publication on acceptance

- support for research data, including large and complex data types

- gold Open Access which fosters wider collaboration and increased citations

- maximum visibility for your research: over $100 \mathrm{M}$ website views per year

At $\mathrm{BMC}$, research is always in progress.

Learn more biomedcentral.com/submissions 